\section{$\underset{\substack{\text { hommes } \\ \text { \& migrations }}}{ }$}

\section{Hommes \& migrations}

Revue française de référence sur les dynamiques

migratoires

1317-1318 | 2017

L'Europe en mouvement

\title{
Les Européens dans les politiques européennes d'aujourd'hui
}

\section{Catherine Wihtol de Wenden}

\section{QpenEdition \\ Journals}

Édition électronique

URL : http://journals.openedition.org/hommesmigrations/3869

DOI : 10.4000/hommesmigrations.3869

ISSN : 2262-3353

Éditeur

Musée national de l'histoire de l'immigration

Édition imprimée

Date de publication : 1 avril 2017

Pagination : $45-51$

ISBN : 978-2-919040-38-4

ISSN : 1142-852X

\section{Référence électronique}

Catherine Wihtol de Wenden, «Les Européens dans les politiques européennes d'aujourd'hui », Hommes \& migrations [En ligne], 1317-1318 | 2017, mis en ligne le 01 avril 2020, consulté le 02 janvier 2020. URL : http://journals.openedition.org/hommesmigrations/3869; DOI : 10.4000/ hommesmigrations.3869 


\title{
LES EUROPÉENS DANS LES POLITIQUES EUROPÉENNES D'AUJOURD'HUI
}

Par CATHERINE WIHTOL DE WENDEN, directrice de recherche au CNRS (Ceri, Sciences Po).

\author{
En mars 2017, le traité de Rome instituant l'Europe \\ économique et politique fête ses soixante ans. Dix millions \\ d'Européens vivent actuellement dans un pays européen \\ autre que le leur, ce qui constitue une mutation importante \\ par rapport aux années antérieures à 2004 où I'on \\ comptait à peine $3 \%$ d'Européens vivant ailleurs que chez \\ eux en Europe. Cette mobilité intra-européenne accrue est due, \\ notamment, à l'expatriation des Polonais et des Roumains, \\ les deux pays ex-communistes ayant la plus nombreuse \\ population et au phénomène récent des «seniors au soleil » \\ où des Européens du Nord et de l'Ouest s'installent à la retraite \\ dans les pays du Sud de l'Europe.
}

En 1957, la question migratoire n'est pas une priorité du traité de Rome bien que plusieurs pays européens éprouvent une pénurie de maind'œuvre dans l'Europe des six (France, Allemagne, Benelux) et qu'un autre pays soit un pays d'émigration, l'Italie. L'essentiel des préoccupations porte sur la libre circulation des marchandises et sur la politique commune du commerce et de l'acier, la Ceca, ancêtre de l'inspiration économique de l'Europe. Ce n'est qu'en 1968 que les travailleurs européens bénéficient de la libre circulation dans l'espace européen avec des droits sociaux qui progressivement s'harmonisent. L'essentiel de ces bénéficiaires sont des Italiens et des frontaliers.
Du traité de Rome à la libre circulation des Européens (1957-2017)

L'entrée successive des pays européens dans la Communauté économique européenne (CEE) puis dans l'Union européenne change la donne. Quand le Royaume-Uni et l'Irlande entrent en 1972, suivis du Danemark, leurs travailleurs peuvent librement travailler en Europe, mais ils sont peu nombreux à le faire. Une autre vague d'entrées survient 
après la fin des dictatures en Grèce, en Espagne et au Portugal, avec des entrées respectives en 1981 et 1986, mais la liberté de circulation, d'installation et de travail ne sera accordée à leurs ressortissants qu'en 1992 pour ces trois pays, considérés comme représentant un risque migratoire pour l'Europe. Souvenons-nous des Portugais venus pour l'essentiel clandestinement en France ayant effectué "O salto ", la traversée irrégulière des Pyrénées avec des passeurs, fuyant la pauvreté et le service militaire de la période

Depuis 1992, le traité de Salazar, à une période où de Maastricht définit la les Algériens, aux termes des citoyenneté européenne

dans son article 8 : une citoyenneté moderne fondée essentiellement sur la liberté de circulation, d'installation et de travail pour les

Européens et assortie de droits civiques locaux pour les résidents européens dans un autre pays que le leur. accords d'Évian, bénéficiaient de la liberté de circulation entre les deux pays jusqu'en 1973. Les Portugais sont toujours la première nationalité en France aujourd'hui.

Entre temps, les accords de Schengen de 1985 sont venus exiger des visas pour les nonEuropéens et définir un passeport européen dès 1986. En revanche, aucune période d'attente ne sera demandée aux Finlandais, Autrichiens et Suédois entrés en 1995, leurs ressortissants ne constituant pas un risque migratoire aux yeux de l'Europe. Depuis 1992, le traité de Maastricht définit la citoyenneté européenne dans son article 8 : une citoyenneté moderne fondée essentiellement sur la liberté de circulation, d'installation et de travail pour les Européens et assortie de droits civiques locaux pour les résidents européens dans un autre pays que le leur.

\section{Une liberté de circuler sans installation}

L'exigence d'une latence entre la période d'entrée et de plein exercice de la citoyenneté européenne est reprise lors de l'entrée des pays de l'Est. Dès la sortie du communisme, en 1991, les pays dits de Visegrad (Tchécoslovaquie, Hongrie, Pologne)

bénéficient de la libre circulation sans liberté d'installation ni de travail en Europe. Des marchés polonais mais aussi russes s'improvisent à Berlin et à Vienne où de faux touristes se font commerçants «à la valise » (tchelnoki) pour vendre tout ce qui peut leur apporter des devises et commencent à travailler au noir, en Allemagne et en Autriche notamment, dans le bâtiment et les services. On trouve sur ces marchés des poupées russes, des insignes de l'armée soviétique qui se retire, des samovars, des chaussures, des réveils, de la fripe... Pour tous ces nouveaux pays européens ainsi que pour les États baltes, l'accès au marché du travail et à la liberté d'installation ne va se mettre en place que selon la volonté des pays d'accueil européens de l'Ouest, selon un système d'attente de 2 + 3 + 2 années : en 2004, lors de l'entrée des nouveaux Européens de l'Est, seuls le Royaume-Uni, l'Irlande et la Suède acceptent leur accès direct à la liberté de travail et d'installation, ce qui crée un afflux de travailleurs polonais dans les îles britanniques alors qu'ils avaient auparavant choisi l'Allemagne comme destination principale. Les autres pays européens préfèrent attendre deux ans en fonction de leur marché du travail, puis trois ans encore s'ils ne s'estiment pas prêts, puis encore deux ans, soit sept ans, qui est la période maximale d'attente imposée. Ainsi, la France choisira la date de 2008, concomitante du pacte européen sur l'immigration et l'asile, pour accorder la liberté d'installation et de travail aux nouveaux Européens de l'Est, qui n'y viendront d'ailleurs pas nombreux. Il s'agit d'une destination seconde pour eux par rapport à l'Allemagne ou au Royaume-Uni. Pour Chypre et Malte, également entrées en 2004, il n'est pas demandé de période d'attente compte tenu de leur faible population et présence migratoires en Europe : la première est plutôt une terre d'accueil, la seconde un lieu de transit et toutes les deux des îles de tourisme.

La question d'une période probatoire pour l'accès à la liberté de travail et d'installation se pose à nouveau lors de l'entrée de la Roumanie et de la Bulgarie dans l'Union européenne, en 2007. Comme leurs autres homologues de l'Est, ils ont bénéficié de 
la liberté de circulation, respectivement en 2000 et 2001. Ces deux pays, les plus pauvres de l'Europe, représentent un risque migratoire aux yeux des pays de l'Union et la Roumanie est le pays le plus peuplé parmi les nouveaux entrants, avec la Pologne. Les Roumains ont d'ailleurs anticipé leur mobilité, commençant dès le début des années 1990 à circuler en Europe pour y effectuer des travaux souvent peu qualifiés (bâtiment, agriculture) ou pour glaner les miettes de la société de consommation occidentale, chez les Roms. Il s'agit alors pour ces derniers de "ne pas se faire prendre dans l'ordinateur » du système d'information Schengen qui les fiche comme indésirables en cas de tentatives d'entrées répétées ou frauduleuses. À partir de leur appartenance à l'Union européenne en 2007, le décompte des sept années d'attente commence, pour s'achever pour tous les pays européens d'accueil en 2014, date de leur accès libre à l'installation et au travail. On voit alors apparaître une migration qualifiée et très qualifiée de médecins, de dentistes, d'ingénieurs, d’architectes, qui coexiste avec la migration de main-d'œuvre. Les pays d'accueil les plus concernés sont ceux qui ont besoin de services liés au tourisme ou à la garde des personnes âgées, comme l'Italie, l'Espagne et le Portugal, où les Roumains figurent parmi les premières nationalités. Mais les Roms, Les Roumains ont d'ailleurs anticipé leur mobilité, commençant dès le début des années 1990 à circuler en Europe pour y effectuer des travaux souvent peu qualifiés (bâtiment, agriculture) ou pour glaner les miettes de la société de consommation occidentale, chez les Roms.

Européens de l'Union ou non, continuent parfois à être reconduits dans leurs pays d'origine d'où ils reviennent, du fait de leur qualité d'Européens. 


\section{De la double absence à la double présence}

L'un des grands enseignements de cette ouverture des frontières en Europe et à l'Est de l'Europe est ce que l'on a appelé la circulation migratoire, la migration d'aller-retour sans nécessairement d'installation longue, à la faveur des facilités de statut, en fonction de la conjoncture économique. Dans ses travaux sur les «badanti », femmes employées à la garde des personnes âgées en Italie, Serge Weber a ainsi montré combien la dis-

Ailleurs et pour les voisins non européens du Sud, la politique des visas est venue durcir les conditions d'entrée, de travail et de séjour pour les anciens pratiquants de la « noria » migratoire, des allers et retours décrits par Abdelmalek Sayad. tance entre l'Italie et la Roumanie ou l'Italie et la Pologne s'estompe quand chacune vit « ici » et « là-bas » du fait des réseaux migratoires transnationaux de colis, d'information avec le téléphone portable et Internet et de transport par bus et par vols low cost. C'est la double présence, qui s'oppose à la "double absence " des migrants du passé, chez eux et en Europe. Mêmes scénario pour les Polonais au Royaume-Uni, cible privilégiée des votants britanniques du Brexit (avec plusieurs morts durant l'été 2016, comme à Harlow) : ceux qui avaient des métiers précaires ou de courte durée sont repartis, tout en s'inquiétant pour leur statut futur si le Royaume-Uni leur imposait des visas. On rencontre aussi ces travailleurs en circulation migratoire permanente entre l'Allemagne et la Pologne (Berlin n'est qu'à $80 \mathrm{~km}$ de la frontière polonaise) et entre l'Autriche et la Moravie (60 km avec Vienne) depuis 25 ans.

Une autre grande leçon de la libre circulation des Européens est la faible substitution des Européens de l'Union aux non-Européens sur le marché du travail, hormis pour les nouveaux Européens de l'Est. Si la figure du «plombier polonais » venu faire concurrence à son homologue de l'Ouest a fait florès, en revanche le projet de substituer l'immigration de travail non européenne par des Européens ne s'est pas réalisé malgré la crise de 2008, car les migrants de l'Est viennent de pays assez peu peuplés, en dehors de la Pologne et de la Roumanie, au profil démographique vieillissant et parfois autosuffisants sur le marché du travail de leurs propres pays.

D'autres pratiques institutionnelles se sont développées, pour anticiper des libertés de circulation futures : ainsi, les Moldaves cherchent à entrer dans les catégories de l'accès à la nationalité roumaine (les deux pays n'en ont fait qu'un dans le passé), et la Hongrie, du fait d'une politique diasporique de la nationalité sur des bases ethnicoculturelles, accorde des passeports hongrois, donc européens, à des Hongrois de l'extérieur, d'Ukraine par exemple.

Ailleurs et pour les voisins non européens du Sud, la politique des visas est venue durcir les conditions d'entrée, de travail et de séjour pour les anciens pratiquants de la "noria » migratoire, des allers et retours décrits par Abdelmalek Sayad. Ceux qui peuvent entrer dans les catégories légales de libre circulation, de liberté d'installation et de travail y procèdent par l'accès à la double nationalité ou par l'utilisation à des fins d'allées et venues des passeports de résidents de longue durée, chez les seniors.

\section{Les Européens dans l'Europe d'aujourd'hui}

Parmi les grandes avancées de l'Europe, mentionnons d'abord les trente ans d'Erasmus, créé en 1987 pour faciliter les échanges d'étudiants à travers leurs programmes universitaires à l'étranger à travers l'Europe. L'idée de former des Européens qualifiés en leur faisant connaître la diversité des cultures, des langues et des enseignements a été unanimement saluée comme un succès, peut-être encore plus civique qu'académique. Elle rend l'Europe plus familière aux Européens comme espace de vie et de travail que par le passé où peu d'Européens s'expatriaient alors qu'ils en avaient le droit, faute de réseaux. L'équivalence des diplômes 
se heurte encore à la résistance des ordres professionnels dans certaines professions, mais la pénurie de certains métiers qualifiés force à accélérer le processus (pour les métiers médicaux). Mais Erasmus ne concerne encore que peu d'étudiants à cause du coût d'expatriation qu'il représente pour les familles les plus modestes et parfois aussi du manque d'intérêt à se projeter dans un univers plus large et plus complexe chez les moins favorisés par le milieu familial. Il contribue pourtant à forger le citoyen européen, un être mobile qui partagerait les mêmes valeurs par-delà les frontières de l'Europe et ferait de celle-ci son cadre de vie et de travail. Mais il risque aussi de faire du citoyen européen le membre d'un club de privilégiés, d'initiés qui partagent la même langue, l'anglais, vit à l'aise dans la mobilité à laquelle les moins qualifiés n'ont pas accès, comme on le constate avec les scores électoraux des eurosceptiques.

Si l'on consulte les données chiffrées des migrations intra-européennes aujourd'hui, on observe que les Roumains et les Polonais figurent parmi les nationalités les plus nombreuses comme migrants internes dans les pays de l'Union et ont donc pleinement mis à profit la liberté de circulation, d'installation et de travail qui leur était offerte. Selon le rapport 2016 de l'OCDE avec des chiffres de 2014, les Roumains sont 1131800 en Italie, 751200 en Espagne, 355300 en Allemagne, $219000 \mathrm{au}$ Royaume-Uni, 73400 en Autriche, 66100 en Belgique, 64800 en France, 31500 au Portugal, 28800 en Grèce et 28600 en Roumanie, soit 2,6 millions dans l'Union européenne. Les Polonais sont 855000 au Royaume-Uni, 674200 en Allemagne, 98700 en Italie, 99600 aux Pays-Bas, 68400 en Belgique, 54300 en Autriche, 48200 en Suède, 32300 au Danemark, 20300 en Grèce, soit près de deux millions dans l'Union. Puis viennent les immigrations des années de croissance, les Portugais au nombre de 509300 en France, 235000 au Royaume-Uni, 130900 en Allemagne, 98400 en Espagne, 92100 au Luxembourg, 42800 en Belgique et, hors Union européenne, 263000 en Suisse, soit 1,4 million en Europe. Les Italiens, au nombre de 574500 en Allemagne, 179100 en
Espagne, 174900 en France, 157000 en Belgique, 19500 au Luxembourg et 308600 en Suisse, soit 1,5 million. Les Espagnols, 146800 en Allemagne, 133400 en France, 79500 en Suisse, 60400 en Belgique, approchent les 400 000. Les Grecs, 328600 en Allemagne, ne sont guère présents en Europe que dans ce pays (16 300 en Belgique).

\section{Des paysages migratoires intra-européens différenciés selon les pays d'accueil}

Si l'on regarde les différents pays européens d'accueil, on constate que chacun de ceux-ci a son paysage migratoire intra-européen : l'Allemagne est de loin le premier pays d'accueil, avec 674000 Polonais, 574500 Italiens, 355300 Roumains, 328600 Grecs, 263300 Croates, 183300 Bulgares, 179800 Autrichiens, 156800 Hongrois, 146800 Espagnols, 144700 Néerlandais et 130900 Portugais. Elle est suivie par le Royaume-Uni, qui compte 855000 Polonais, 379000 Irlandais, 235000 Portugais, 219000 Roumains, 192000 Lituaniens, 189000 Français, 167000

Espagnols, 119000 Allemands et 117000 Lettons. Puis vient la France, avec 509300 Portugais, 174900 Italiens, 156400 Britanniques, 133400 Espagnols, 95100 Belges, 93100 Allemands et 64800 Roumains, tout en étant peu concernée par les nouveaux migrants de l'Est européen. L'Espagne accueille à la fois de nouveaux migrants de Si l'on consulte les données chiffrées des migrations intra-européennes aujourd'hui, on observe que les Roumains et les Polonais figurent parmi les nationalités les plus nombreuses comme migrants internes dans les pays de l'Union et ont donc pleinement mis à profit la liberté de circulation, d'installation et de travail qui leur était offerte.

l'Est et des seniors au soleil, avec 751200 Roumains, 282100 Britanniques, 179100 Italiens, 141900 Bulgares, 130500 Allemands, 99000 Français et 98400 Portugais. L'Italie n'est concernée, mais massivement, que par les Roumains (1 131 800) et les Polonais (97 700), et aussi par d'autres Européens qui ne font pas par- 
Étudiants Erasmus, sur les marches de l'Église Santa Engrácia, Lisbonne. @ D. R.

tie de l'Union, les Albanais, ceux-ci étant aussi massivement présents en Grèce. La Belgique, avec 159400 Français, 157000 Italiens, 149800 Néerlandais, 68400 Polonais, 66100 Roumains, 60400 Espagnols, 42800 Portugais, 29000 Bulgares, 24000 Britanniques et 16300 Grecs, est aussi fortement concernée par les migrations intra-européennes. Il en va de même en Autriche qui compte une importante population de l'est, outre les 170500 Allemands : 73400 Roumains, 66500 Croates, 54900 Hongrois, 54300 Polonais, 32100 Slovènes, 22500 Italiens, 19600 Bulgares, 13500 Slovaques. Enfin, hors de l'Union européenne, la Suisse concentre une importante population intra-européenne avec 308600 Italiens, 298600 Allemands, 263000 Portugais, 116800 Français, 79500 Espagnols, 41100 Bri- tanniques, 40000 Autrichiens, 44000 Polonais et 20500 Néerlandais. Ce sont les pays situés le long des frontières de l'ancien rideau de fer qui abritent le plus de migrants de l'Est européen, Grèce incluse (43 300 Bulgares, 28800 Roumains et 20300 Polonais), mais où les Albanais voisins forment plus de $60 \%$ des migrants.

Les seniors au soleil, qui s'installent à demeure dans un autre pays européen plus ensoleillé que le leur (France, Espagne, Portugal, Malte, Italie), marquent une tendance récente : on compte ainsi 282100 Britanniques en Espagne, 156400 Britanniques en France (dans le Sud-Ouest notamment), 16600 au Portugal, soit près de 500000 en Europe. Les Allemands sont 130500 en Espagne et 93400 en France. Tous ne viennent pas que pour s'y reposer : ils y créent parfois une activité 
à distance (avec Internet) ou de proximité (restauration de maisons anciennes, boutiques de brocante, restaurants ruraux...). Enfin, le chômage de qualifiés dans le Sud de l'Europe depuis la crise de 2008 a aussi provoqué une migration vers les pays du Nord de l'Europe comme les 189000 Français répertoriés au Royaume-Uni et autres Italiens, Espagnols, Portugais et Grecs dans les pays nordiques.

La migration de proximité (frontaliers, migrations de voisinage, migrations pour raisons fiscales) tient aussi une part non négligeable dans la migration intra-européenne, comme les 379000 Irlandais au Royaume-Uni et les 114900 Britanniques en Irlande, les 159400 Français en Belgique, 39400 au Luxembourg, les 116800 Français en Suisse, les Italiens (308 600) et les Allemands (298 600) dans ce dernier pays, les 170500 Allemands en Autriche, les 71800 Allemands aux Pays-Bas, les 144700 Néerlandais en Allemagne, les 43300 Bulgares en Grèce, les 28600 Roumains en Hongrie, les 40000 Autrichiens en Suisse.

\section{Conclusion}

Tout cela donne une image d'une Europe mosaïque, bien que ces migrations intra-européennes ne représentent qu'à peine 9 millions de personnes sur 42 millions de migrants internationaux dans une Europe de 506 millions d'habitants, Suisse exclue. De surcroît, il s'agit d'un chiffre incertain car une partie des Européens ne se font pas enregistrer quand ils migrent dans un autre pays européen. Il y a donc encore beaucoup à faire pour que la migration intra-européenne fasse partie des pratiques de vie courantes des Européens et qu'ils s'approprient pleinement l'espace dans lequel ils sont amenés à exercer leur travail et la citoyenneté européenne. On peut s'étonner que le chômage des qualifiés et des non-qualifiés, la circulation de l'information et les multiples maillages transnationaux intra-européens existants (jumelages de villes, programmes scolaires, Erasmus, échanges linguistiques, amitié franco-allemande) n'aient pas provoqué davantage de mobilité : plus on est qualifié, plus on est mobile dans un espace plurinational facilement maitrisé car devenu familier, moins on est qualifié, moins on est mobile car le coût de cette mobilité apparaît très élevé, faute de réseaux. Il faut aussi signaler des formes différenciées de présence des Européens au sein des pays de l'Union européenne. Les couples migratoires, comme les Roumains en Italie et en Espagne, demeurent beaucoup moins nombreux ailleurs. Les migrations des Italiens et des Portugais adoptent des formes quasi-diasporiques. Ils sont répartis dans plusieurs pays européens avec des liens parfois entretenus par le pays d'origine, l'action de leurs associations culturelles. Les ressortissants des pays de l'Est relèvent quant à eux d'une forme de «saupoudrage » : ils s'installent dans plusieurs pays européens, sans nécessairement créer des liens entre eux, car ils communiquent plutôt avec leur pays d'origine. I

\section{Bibliographie}

Dana Diminescu, Visibles mais peu nombreux. Les migrations roumaines, Paris, éd. de la MSH, 2003. Patrick Dollat, La liberté de circulation des Européens, Paris, La Documentation française, 2005. OCDE, SOPEMI, Perspectives des migrations internationales 2016, Paris, OCDE, 2016.

Anne de Tinguy, La grande migration, Paris, Plon, 2004.

Serge Weber, Les migrations dans la nouvelle Europe, Paris, Le Félin, 2007.

__-, "Des chemins qui mènent à Rome ", thèse de géographie, Paris, Université de Paris I, 2004.

Catherine Wihtol de Wenden, La citoyenneté européenne, Paris, Presses de Sciences Po, 1997.

- Migrations. La nouvelle donne, Paris, éd. de la FMSH, 2016

—-, Atlas des migrations. Un équilibre mondial à inventer, Paris, Autrement, 4e édition, 2016.

—_, L'immigration, Paris, Eyrolles, 2016. 